\title{
Effect of Short Foot Exercises on Patients with Flexible Flat Foot: A Pre-Post Experimental Study
}

\author{
Abha Khisty ${ }^{1}$, Rutuja Kulkarni ${ }^{2}$, Pallavi Desai ${ }^{3}$, Tushar J. Palekar ${ }^{4}$ \\ ${ }^{1}$ Assistant Professor, ${ }^{2}$ Intern, ${ }^{3}$ Professor, ${ }^{4}$ Principal and Professor, Dr. D.Y. Patil College of Physiotherapy, Pune \\ Corresponding Author: Abha Khisty
}

DOI: https://doi.org/10.52403/ijhsr.20220115

\begin{abstract}
Background: When one malleolus was lower than the other malleoli and calcaneal eversion with depressed medial longitudinal arch, pronated sub-talar joint and calcaneus assuming a valgus position underweight bearing condition, known as flatfoot or Pes Planus. Generally, 20-30 \% of the population between the age group 18-25 had the characteristic of a Pes Planus also known as flat foot. The intent of this experiment was to investigate the effect of 4 week Short Foot Exercise Program on intrinsic foot muscles leading to improvement in performance grade classified as from fair to good.

Method: An experimental study conducted at Dr. D.Y. Patil College of physiotherapy, Pimpri, Pune comprising of 30 individuals using Simple Random sampling. Participants were divided into two groups 15 participant each Group A(15) was Conventional Therapy and Group B(15) was Short Foot Exercise progression. The subjects were each given a consent form. The purpose of the study was explained to all the participants and an informed consent was taken from each subject. Navicular Drop Test ( $\mathrm{r}=0.92)$ was used as an Outcome Measure.

Results: There was clinically and statistically significant difference in Group B(Short Foot Exercise Progression) than Group A(Conventional Therapy) ( $\mathrm{p}<0.005$ and mean of group A more than B) pre and post $2^{\text {nd }}$ Week treatment and Post $4^{\text {th }}$ week Treatment.

Conclusion: The Short Foot Exercise is more effective in improving Medial Longitudinal Arch intrinsic muscle activation as compare to Conventional Group. The 4 week Short foot exercise program improved foot intrinsic muscle activity in people with flat foot. However, Group B that is Short Foot Exercises shows better improvement in medial longitudinal arch than Group A That is Conventional Group.
\end{abstract}

Key Words: Pes Planus, Flat Foot, Navicular Drop Test, Foot biomechanics, 4 week Short Foot Exercise Progression.

\section{INTRODUCTION}

The ankle and foot were the most distal segment in the lower extremity and it occupies $5 \%$ of area of the human body. ${ }^{1}$

The foot ankle complex includes bone, muscle and ligament which support the medial longitudinal arch which gives shape, strength and stability to the joint. ${ }^{2}$

The foot ankle complex includes Sub- talar joint or talo-calcanean joint, talocalcaneonavicular joint and calcaneocuboid joint which were the main inter-tarsal joints. While cuneonavicular joint, cuboideonavicular joint, intercuneiform and cuneocuboid joints were the smaller inter-tarsal joints. ${ }^{3}$

The foot has two primary functions at the time of locomotion i.e. Stance and propulsion. The foot was flexible to absorb the energy which was generated from the ground. The intrinsic foot muscle provides firmness and stability for the propulsion while flexibility for shock absorption and attenuation forces. Improper function of the intrinsic foot muscles caused excessive pronated foot. ${ }^{4}$ 
The foot had pronated as well as supinated structure, muscles helps to maintaining the stability of the support base. Pronation of sub talar joint caused excessive flat feet. 200 of pronation (eversion) and 300 of supination (inversion) was the normal degree of freedom for ankle joint. ${ }^{5}$

When one malleolus was lower than the other malleoli and calcaneal eversion with depressed medial longitudinal arch, pronated sub-talar joint and calcaneus assuming a valgus position underweight bearing condition, known as flatfoot or Pes Planus. It begins in childhood and continues in to adulthood. ${ }^{6}$ There were two different types of flat foot; rigid or congenital and acquired or flexible

Rigid or Congenital Flat foot which was very rare. In this type, calcaneus was in valgus position, mid-tarsal was in pronation, and the navicular was displaced dorsally and laterally on the talus. Second type was Acquired or Flexible Flatfoot, in which the foot is mobile. The term 'flexible' means while standing or during weight bearing the foot was flat. ${ }^{7}$

Generally, 20-30 \% of the age group between 18-25 had the flat foot. ${ }^{2}$

Intrinsic foot muscle strengthening help to prevent pronation related injuries and integrity of the medial longitudinal arch.

This helps in reducing navicular drop scores and plantar arch index scores. Reduction in both these scores helps in improving flat foot. Hence the previous article on effectiveness of gluteal muscle strengthening on flat feet proves that strengthening of intrinsic muscle helps in improving flat foot. ${ }^{8}$

Flexible flatfeet may relate to secondary injuries like ankle sprains, plantar fasciitis also deformities include hallux valgus. $^{9}$

The flat feet deformity was induced when the medial longitudinal arch had decreased because the arch cannot maintain and caused excessively pronated feet compared to normal feet and weight bearing was shifted inward to compress medial longitudinal arch. ${ }^{10}$

The flexible flat foot was commonly seen in the adults, the management and awareness of the same was necessary. These exercises aid in biomechanically correcting the medial longitudinal arch of the foot therefore correcting the flexible flat foot deformity.

Several Studies have proved that effect of Short Foot Exercises in relation to sensorimotor training for subject with Neutral feet.

There are many articles which have been proven effectiveness on other foot conditions but there are very limited studies conducted on flat feet and effect of only intrinsic exercises on flat feet using navicular Drop Test as an outcome measure.

4 week Short Foot Exercise Program will help to increase the performance grade of foot intrinsic muscle from fair to good.

Therefore, this study was helpful in determining the effect of foot intrinsic strengthening exercises and short foot exercise in subjects with flat feet.

\section{MATERIALS AND METHODOLOGY}

Ethical approval for conducting the study was obtained from institutional ethics committee. Written informed consent was obtained from the participants. Individuals who fulfilled the inclusion criteria were included in the study. Participants were informed about the purpose of the study. The treatment protocol and its duration were explained to the patients. Patients from age group 18 to 30 with flexible flat feet were included. Patients having arthritis of knee, Diabetic neuropathy, recent foot and ankle surgery leg length discrepancy and pathology that affect feet, knee, hips and spine were excluded.

Procedure and data collection: 30 individuals with Flat Feet were selected in the study fulfilling the inclusion criteria (Navicular Drop Test $>10 \mathrm{~mm}$

\section{Outcome measure: Navicular drop Test:}

In navicular drop test the subject were placed in a sitting position with their 
feet flat on a firm surface and with knees flexed to 90 degrees and ankle joint in neutral position. The most prominent point of the navicular tubercle while maintaining sub-talar neutral position was identified and marked with a pen. Now place the card on the inner aspect of the hind-foot from the floor in a vertical position passing the navicular bone. Finally, the difference between the original heights of the navicular tuberosity was assessed with a measuring tape rendering the navicular drop amount in millimeters. $^{2}$

The procedure of Conventional Therapy and Short Foot Exercise was explained to the patients.

Total duration of the treatment: 4weeks, 6 session/week, once a day

Time: $10 \mathrm{~min} / \mathrm{session}$

Total number of sessions: 24

\section{Conventional Group (Group A):}

This group consisted of candidates who followed an intrinsic muscle strengthening protocol-

i. Heel raises: Patient was in standing position and has to perform heel raises 20 times. (without shoes)

ii. TA Stretching: The patient was taken in supine position Researcher was positioned to side of the patient and applied a passive stretch leading to 30 sec hold and was repeated 5 times each session. Toe Spreading exercises: The patient was in sitting or standing position and shall be instructed to stabilize the ball of the foot on the ground while lifting and spreading out the toes, Frequency: 20 times, intensity: 5 sec holds, type of contraction: isometric contraction.

iii. Toe extension exercises: The patient was in sitting or standing position and was instructed to lift the big toe against the floor. Frequency: 20 times, intensity: 5 sec holds, type of contraction: isometric contraction.

\section{Experimental Group / Short Foot Exercise (Group B):}

This group was consisting of candidates which were following an intrinsic muscle strengthening protocol.

\section{Short foot exercises:}

i. The candidates placed the foot flat on the ground, and draw the metatarsals inwards creating an arch with progression. Frequency: 20 times, intensity: 5 sec holds, type of contraction: isometric contraction.

\begin{tabular}{|l|l|l|l|l|}
\hline $\begin{array}{l}\text { Short Foot Exercise } \\
\text { Progression }\end{array}$ & $\mathbf{1}^{\text {st }}$ Week & $\mathbf{2}^{\text {nd }}$ Week & $\mathbf{3}^{\text {rd }}$ Week & $\mathbf{4}^{\text {th }}$ Week \\
\hline Positions & Sitting position & Standing on Both Legs & Standing on one Leg & Standing on an Unstable board on one leg \\
\hline \multirow{2}{*}{$\begin{array}{l}5 \text { sets per day } \\
\text { Repetitions }\end{array}$} & $\begin{array}{l} \\
5 \text { min restitions between each set }\end{array}$ \\
\hline
\end{tabular}

\section{RESULT}

An Experimental study was conducted on 30 patients. Shapiro-wilk test was applied to see the normal distribution, $\mathrm{p}>0.05$ so the data was normally distributed, hence the parametric tests were applied. For within group analysis paired test was applied whereas for between comparisons unpaired t test was applied and for between group analysis unpaired $t$ tests were applied.

Graph no. 1 indicates the difference between navicular drop test in pre-treatment with mean 12.4 standard deviation \pm 0.82 and post 2nd week treatment of Group B with mean 11.53 standard Deviation \pm 0.63 with $\mathrm{p}<0.05$.

Graph no. 2 indicates the difference between navicular drop test in pre-treatment with mean 12.4 standard deviation 0.82 and post 4th week treatment of Group B with mean 10.53 standard Deviation $0.63 \mathrm{p}<0.05$.

After intervention of navicular drop test post treatment showed significant decrease in Group B than Group A. It showed clinically and statistically significant difference in Group $B$ than Group $\mathrm{A}(\mathrm{p}<0.005$ and mean of group $\mathrm{A}$ more than B) pre and post 2nd Week treatment and Post 4th week Treatment. 


\section{DISCUSSION}

Flat foot is a condition in which the medial longitudinal arch is depressed, subtalar joint is pronated and the calcaneus is in valgus position under weight-bearing.

The incidence of the flat foot has been reported to be $11.25 \%$ in a population of 18-25 years old Students. Since many studies set the criterion for the flat foot as $10 \mathrm{~mm}$ or greater navicular drop test.

The aim of my study was to compare the effect of short foot exercise and conventional therapy in young adults with flat feet. After intervention the study showed the significant results.

In the previous study in case of flat foot, to improve this condition to correct Medial Longitudinal Arch the intervention method was include Short Foot Exercise for strengthening the intrinsic muscles and extrinsic muscles of the foot for six weeks. ${ }^{1}$

Toes-spread-out exercise, first-toeextension exercise, and second- to fifth-toeextension exercise each caused activation of all of the intrinsic plantar foot muscles and can be recommended as a means of exercising these muscles.

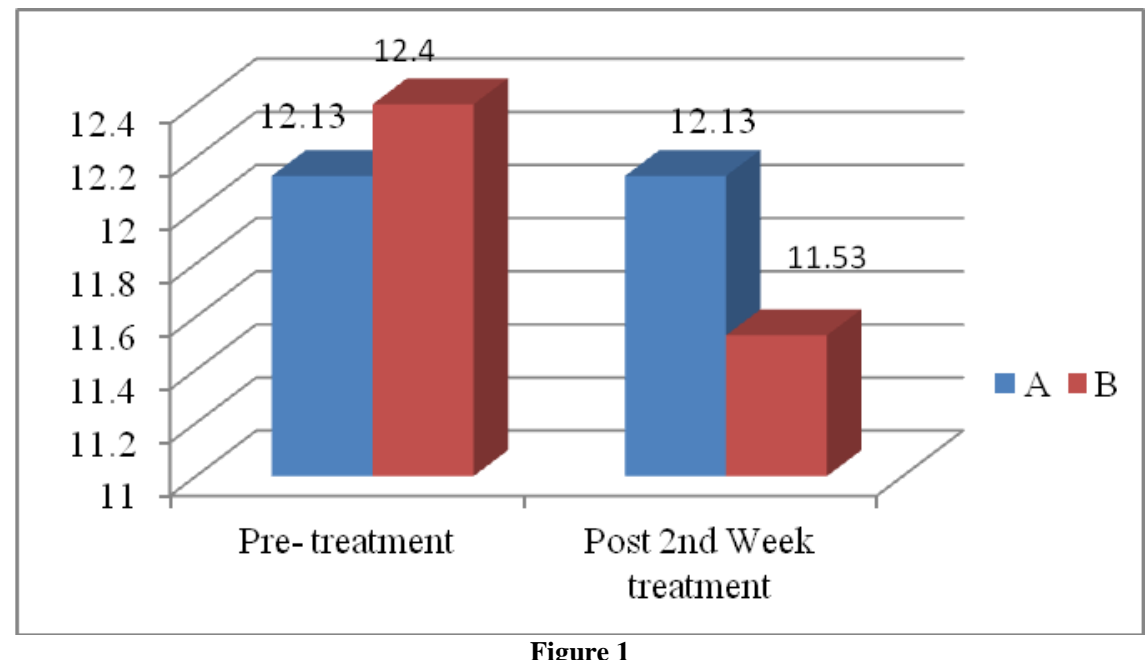

INTERPRETATION: The above Graph shows the Difference between Navicular Drop Test in Pre - Treatment and Post 2nd week treatment of group A (Control) and group B (Experimental). It shows significant difference in Post 2nd week than pre- treatment with $\mathrm{p}<0.05$.

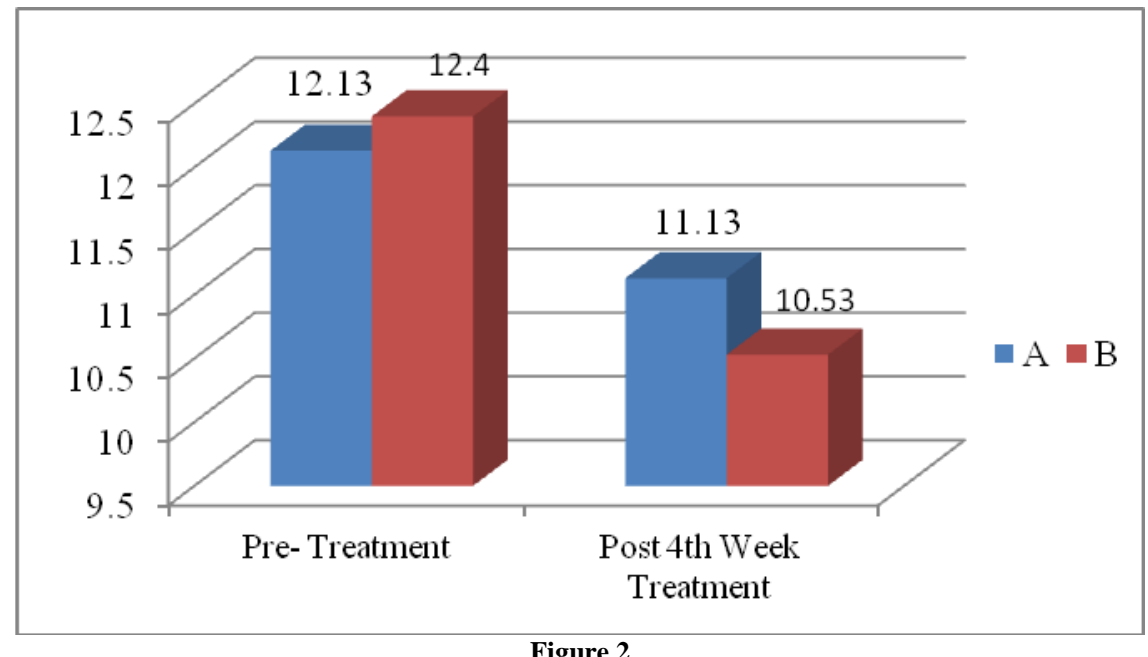

INTERPRETATION: The above Graph shows the Difference between Navicular Drop Test in Pre - Treatment and Post 4th week treatment of group A (Control) and group B (Experimental). It shows significant difference in Post 4th week than pre- treatment with $\mathrm{p}<0.05$. 
Graph 1 shows significant changes in mean difference of pre-treatment and post 2nd week treatment of Group A (control) and Group B (experimental).

Graph 2 showed significant changes in the mean difference of pre- treatment and post 4th week treatment of Group A (control) and Group B (experimental).

In other study they concluded that the short foot exercise is more effective than the towel-curl exercise to training the intrinsic foot muscles to maintained the height of medial longitudinal arch. ${ }^{4}$

The previous study demonstrated that a 4-week Short Foot Exercise (SFE) program improved activation of foot intrinsic muscles and static and dynamic balance. These findings prove that SFE can be a clinically significant program for individuals with Flat Foot as well as those with Neutral Foot. ${ }^{9}$

This study demonstrated that 4 week Short Foot Exercise progression has positive effect on Flat Foot; it helps to strengthen the intrinsic foot muscles with changing the position and different Base of support.

\section{CONCLUSION}

The Short Foot Exercise program is more effective in improving Medial Longitudinal Arch strength (as seen in group B/ experimental group) as compared to Conventional Group. In general, the 4 week foot exercise program improved foot intrinsic muscle activity in people with flat foot. However Short Foot Exercises group showed statistically significant difference compared to Group Conventional Group.

Acknowledgement: None

\section{Conflict of Interest: None}

\section{Source of Funding: None}

\section{Ethical Approval: Approved}

\section{REFERENCES}

1. Kim EK, Kim JS. The effects of short foot exercises and arch support insoles on improvement in the medial longitudinal arch and dynamic balance of flexible flatfoot patients. J Phys Ther Sci. 2016;28(11): 3136-3139. doi:10.1589/jpts.28.3136.

2. Namrata Sojitra, Sheetal Patel., A Study To Compare Dynamic Balance Between Individuals With Flat Feet And Individuals With Normal Arched Feet Using Y- Balance Test - An Observational Study. Indian Journal Of Physical Therapy Volume 5 Issue 1 (January - July 2017).

3. B D Chaurasia Human Anatomy volume 2. 6th Ed. 2013 page no. 151.

4. Scott K. Lynn, Ricardo A. Padilla, and Kavin K.W. Tsang., Differences In StaticAnd Dynamic-Balance Task Performance After 4 Weeks Of Intrinsic-Foot-Muscle Training: The Short-Foot Exercise Versus The Towel-Curl Exercise. Journal of Sport Rehabilitation, 2012, 21, 327-333.

5. Dong-chul Moon, PT, MS1, KyoungKiM, $\mathrm{PT}$, PhD1, Su-Kyoung lee, PT, PhD2., Immediate Effect of Short-foot Exercise on Dynamic Balance of Subjects with Excessively Pronated Feet. J. Phys. Ther. Sci. 26: 117-119, 2014.

6. Pamela K. Levangie, Cynthia C. Norkin. Joint Structure And Function. 5th Ed. Page452.

7. David J. Magee. Orthopedic Physical Assessment. 6th Ed. 2014 Page 912.

8. Pooja Mulchandani, Trupti Warude, Amrutkuvar Pawar., Effectiveness Of Gluteal Muscle Strengthening On Flat Foot. Asian J Pharm Clin Res, Vol 10, Issue 6, 2017, 219-221.

9. Jeongah Kim., Muscle Activity and Balance in People with and without Flexible Flatfeet Following a Short Foot Exercise Program. The Graduate School Yonsei University Department of Physical Therapy 2014.

10. Jeong-ah Kima, One-bin Lima, ChunghwiYib, Difference In Static And Dynamic Stability Between flexible flatfeet And neutral feet. GAIPOS-4376; No. Of Pages5

11. Gooding TM, Feger MA, Hart JM, Hertel J. Intrinsic Foot Muscle Activation During Specific Exercises: A T2 Time Magnetic Resonance Imaging Study. J Athl Train. 2016 Aug;51(8):644-650. doi: 10.4085/1062-6050-51.10.07. Epub 2016 Oct 3. PMID: 27690528; PMCID: PMC5094843.

12. Moon-Hwan Kima, Oh-Yun Kwon b, SiHyun Kimc and Do-Young Jungd,*. 
Abha Khisty et.al. Effect of short foot exercises on patients with flexible flat foot: a pre-post experimental study.

Comparison of muscle activities of abductor hallucis and adductor hallucis between the short foot and toe-spread-out exercises in subjects with mild hallux valgus. Journal of Back and Musculoskeletal Rehabilitation 26 (2013) 163-168.

13. Unver B, Erdem EU, Akbas E. Effects of Short-Foot Exercises on Foot Posture, Pain, Disability, and Plantar Pressure in Pes Planus. J Sport Rehabil. 2019 Oct
18;29(4):436-440. doi: 10.1123/jsr.20180363. PMID: 30860412.

How to cite this article: Khisty A, Kulkarni R, Desai P et.al. Effect of short foot exercises on patients with flexible flat foot: a pre-post experimental study. Int J Health Sci Res. 2022; 12(1):105-110. DOI: https://doi.org/10.52403 /ijhsr.20220115 